\title{
Study on Approaches of Noise Cancellation in GSM Communication Channel
}

\author{
Rekha N \\ Asst. Prof: Dept of Telecommunication Engg., \\ K.S Institute of Technology \\ Bangalore, India
}

\author{
Fathima Jabeen, PhD \\ Prof. and Head Dept of Electronics \& \\ Communication KSSEM, Bangalore, India
}

\begin{abstract}
With the increasing base of mobile phone users globally, the need of providing quality voice calls has become one of the prominent need of the telecommunication. GSM has a wide customer base where various instances of noisy calls has been witnessed. Hence, the evolution of noise cancellation technique has come as a boon to eliminate noise from the sensitive and critical signals for the superior quality of services towards various commercial communication systems over GSM. Although there has been a bunch of research work conducted for noise cancellation, but very few are evident in the field of GSM communication system. This paper discusses the cause of noise in GSM along with various causes and effect. It also frequently adopted technique with various noise quality measuring practices. Finally, it discusses some of the prior studies witnessed to address the similar issues in the past.
\end{abstract}

\section{Keywords}

Echo, GSM, Interference, Noise Cancellation

\section{INTRODUCTION}

In the most recent decade the utilization of cellular telephones expanded significantly and with it's the quantity of sound recordings which are polluted with another sort of audio sound disturbances. This noise in its nature leads a wide range of harsh sound along with Signal-to-Noise Ratio (SNR) is negative. Speech signal enhancement in noisy situations, for example, in vehicles, trains, street roads and at public venues, enhances the quality and clarity of speech signal. Reduction in noise gives advantages in a wide range of uses, like cellular telephones, hands-free telephones, video chatting and an automated secure speech communication services. In this manner, the comprehensibility and the audibility is diminished. Interference noise cancelation for GSM frameworks has received significant attention to expanding the framework limit. Keeping in mind the end goal to reduce this noise present in sound signal, a few algorithms have been designed.
The fundamental issue of hands-free telephony is the acoustic echo: at the near-end the amplifier signal is collected by the receiver and transmitted back to the far-end users. The acoustic echo is particularly disturbing when huge amount of data transmission delays happen. Consequently, the nature of the cellular telephone environment with its long transmission delays (e.g., the GSM framework has a round trip delay of around $180 \mathrm{~ms}$ ) makes the acoustic's cancelation echo a basic issue. Another issue emerges when a hands free phone is utilized as a part of a noisy environment, for instance, in a vehicle. Indeed, even background noise levels can prompt low SNRs. Normally, the SNR is around $20 \mathrm{~dB}$ lower contrasted with utilizing a handset. Hence, the reason, the removal of background noise signal to be transmitted is very desirable, particularly when a low to medium bit rate voice codec is used for transmission. These codecs are not straightforward regarding background noise and the voice quality may be significantly corrupted in the vicinity of strong background noise.

Initially work was done by Rosengern and Nilson [1] for "honey bee commotion" diminishment in cellular telephones. Due to periodically nature, the noise has peaks at different of main frequency which is approximately $217 \mathrm{~Hz}$. along these lines the author proposed the use of fixed score channel for the noise removal. Besides, an algorithm basically depends on matched-filtering or correlation indicates great noise removal capacities. Unfortunately, this scheme relies on upon from the earlier information, which is accessible in a cellular telephone however not in a legal work case. The conventional noise cancellation technique is the Wiener filter, which was modified the size of frequency spectrum range of the noisy input speech signal in extent to an estimate of the signal to noise ratio at every frequency. The Wiener filters requires finding of power spectra. De-noising signal improves the intelligibility and quality of secure speech communication in very noisy environs and also decreases the weakness present in communications system. 


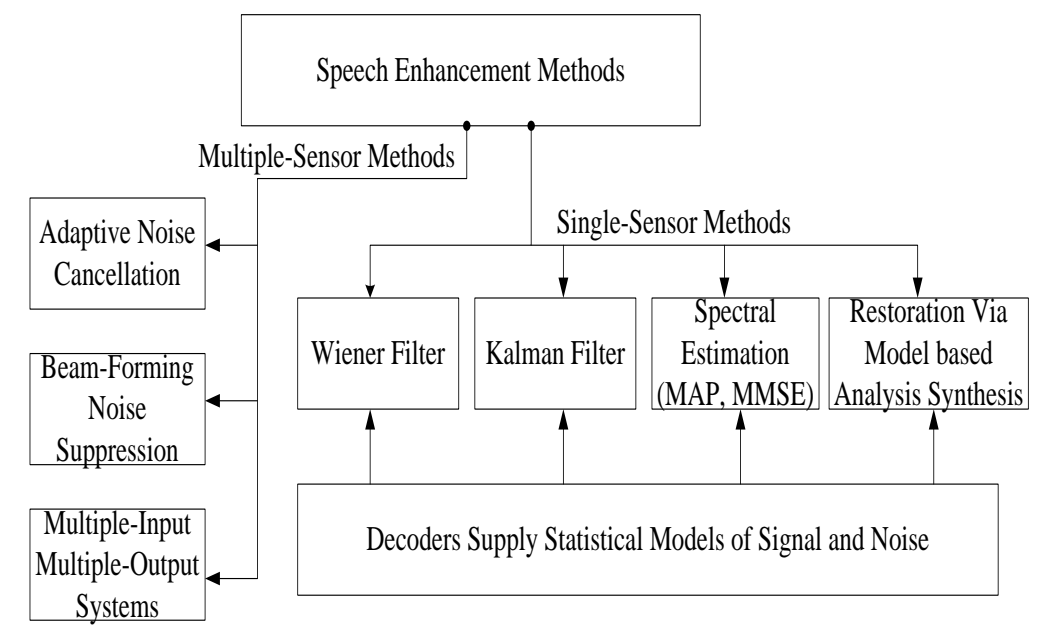

Fig.1. A Classification of Speech Enhancement Techniques

Figure.1 shows a classification of the noise reduction techniques for speech signal used for single input and multiple-input circumstances. In order to succeed the better noise-removal performance, where possible, the benefits of the signal processing approaches established for single-input noise destruction and multiple-inputs noise elimination classifications are combined. This issue was tended to by Harrison [2]. He gave another new method taking into account an adaptive noise canceller to remove noise. This methodology appears to function admirably, for stationary situations, yet falls flat for genuine recordings, because of the variance of pace in little attractive recording gadgets. With a specific goal to comprehend and investigate the understanding the inside of GSM burst, the proposed system will inspect it from a course to a fine scale. Figure 2 shows a typical recording signal with a GSM cell phone close to the amplifier. In this first case the signal was digitized directly, so no center recording system was not used.

This segment of about $3 \mathrm{~s}$ length has some dynamic points (e.g. 0 to $0.2 \mathrm{~s}$ ) and some unmoving parts $(0.6 \mathrm{~s}$ to $1.2 \mathrm{~s})$. The input signal has low power, in this way, the SNR for this example is low. The unmoving parts are made by the Voice Activity Location (VAD) segment inside the GSM framework. In the event that the VAD distinguishes times of silence, the radio's transmission signal diminished to give more transfer speed to other cell telephone users. Besides, (which can't be found in this illustration), there is a power control for the transmitter and in some recording device there will be a programmed addition control framework [3]. Both frameworks will change the adequacy of the GSM signal after some time. As a first result of this investigation proposed system can't expect long term stationary for the burst signal.

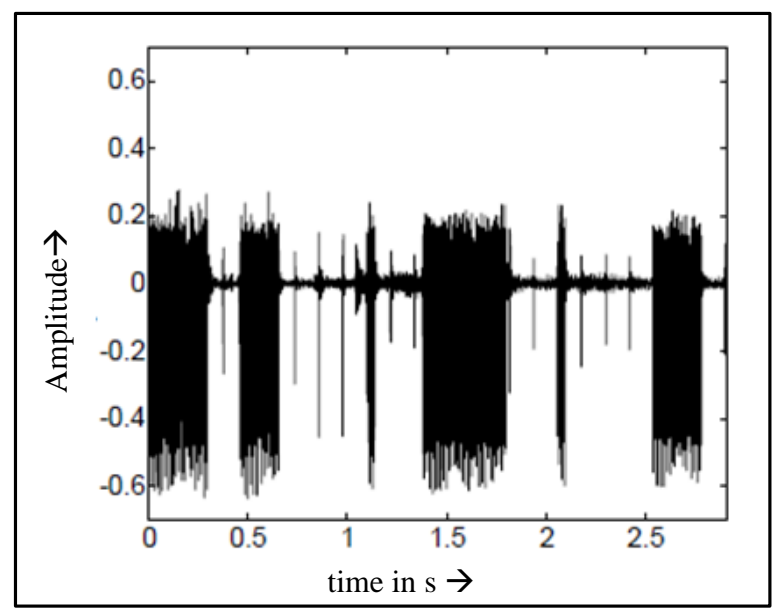

Fig.2. Large Structure of a Speech Signal Contaminated with GSM Bursts

Figure 3 demonstrates a zoomed view of the dynamic area toward the beginning of file. This prompts two assumptions. Indeed, even short-time stationary can't be expected because of the idle edge and GSM burst is example of an additive disturbance.

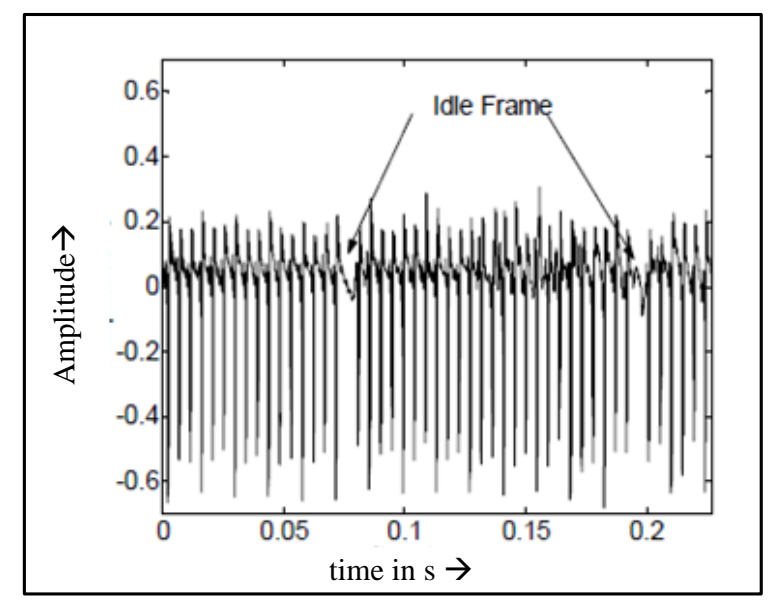

Fig.3. Structure of the GSM Signal with Idle Frame

Then again, this first model is not generally correct. Particularly, if transmission systems with constrained 
dynamics are utilized, the GSM-burst can bring about overloading, which is cut-out for digital signals and magnetic tapes immersion for analog media.

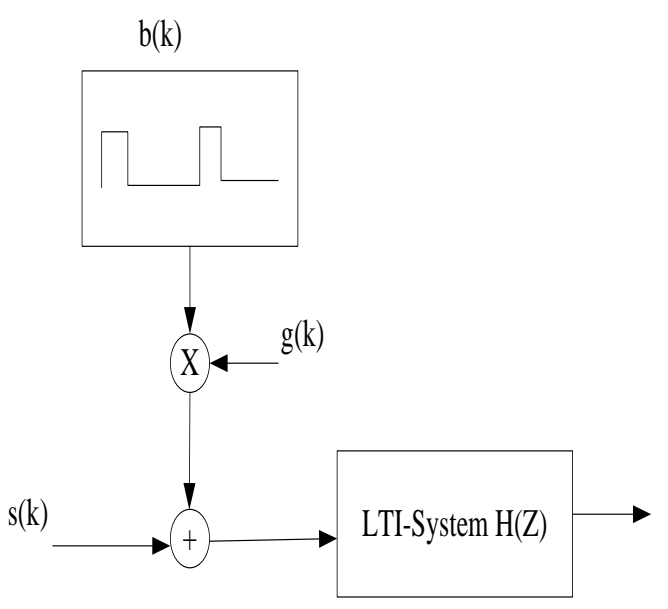

Fig.4. Additive Signal Model

On the other hand, proposed work will concentrate on these two models. It is imperative to comprehend that the GSM burst structure is a period space issue and along these lines a period area methodology should be utilized. Frequency-space methodologies like notch filtering will separate because of the jumble of issue and arrangement.

The proposed paper reviews some of the standard approaches that are responsible for performing noise cancellation in GSM communication channel. Then, it give the issues of noise present in GSM transmission methods. Section 2 briefly elaborates about the origination of the noise in GSM followed by Section 3 that discusses about the various issues encountered in this area. Section 4 illustrates standard techniques accompanied by GSM noise measurement practices in Section 5. Section 6 discusses about the prior research work in the same field followed by conclusion in Section 7.

\section{ORIGINATION OF NOISE IN GSM}

Noise, fading and the ratio of bandwidth are the important factor that, the speed and capacity of speech communication on wireless channels. Most of the developers and research are designed a new generation of mobile communication designs aim to increases the spectral efficiency and it is defined as number of bits per second per Hertz of the bandwidth of communication channel. The main objective is limited resources in modern communication systems are bandwidth and on board battery power. The available bandwidth is limited by the need to share the finite radio frequency channels among many different mobile users and many different applications.

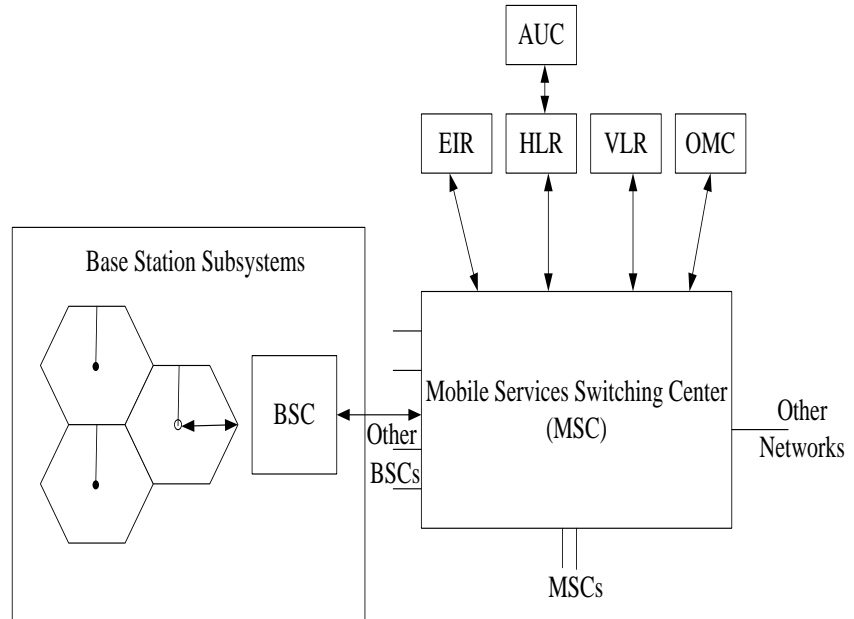

Fig.5. Illustration of a basic GSM Mobile Phone Network

Figure.5 shows the basic network architecture GSM cellular mobile phone system. Noise can be defending as an unwanted signal that interferes with the communication or measure of another signal. A noise itself is a signal that conveys information regarding the source of the noise. Noise and distortion are the main factors limiting the capacity of data transmission in telecommunication and accuracy in signal measurement systems. depending on its sources, a noise can be classified into a number of categories are: acoustic noise, thermal noise, short noise, electromagnetic noise, electrostatic noise, channel distortion, echo, fading and processing noise. Depending on its frequency spectrum or time characteristics, a noise process can be further categorized as White noise, band limited noise, narrowband noise, colored noise, impulsive noise and transient noise pulses.

Single-channel noise reduction is a quite difficult challenge, since the speech and the noise are mixed in the same channel. A large portion of these methods depend on familiar family of speech improvement algorithms: like Short time spectral attenuation method. They attempt to find short time spectral magnitude of speech using attenuation to Fourier transformation coefficients of STFT coefficients of noisy speech in relation to SNR. The phase of the noisy speech is not processed, based on the assumption that phase distortion is not perceived by the human ear [6]. These techniques can be classified into three types:

- $\quad$ Power spectral subtraction [7] consists in subtracting an estimate of the noise power spectral density from the power spectral density of the microphone signal;

- Spectral amplitude subtraction [8], which consists of subtracting from amplitude the estimate of the amplitude of the spectral noise component.

- Direct implementation of the Wiener solution by an open-loop filter of the microphone signal, which consists of minimizing the root-mean-square (RMS) error.

However, it has been widely reported that the noise remaining after the processing has a very unnatural disturbing quality, produced by sinusoidal segments with a random frequencies that will go in short time frames. This artifact is known as the "musical noise" phenomenon. Different alterations of the fundamental noise suppression have been proposed to overcome this issue magnitude averaging [8], overestimation 
of the noise power and introduction of a spectral floor [9], soft-decision noise suppression filtering [10], optimal MMSE estimation of the short-time spectral amplitude [11], a decision- directed approach [12],

Nonlinear spectral subtraction [13], introduction of masking properties of the human auditory system [14], and morphological-based spectral constraints [15].

\section{VISUALIZING THE ISSUE}

In GSM mobile telephony it is a common problem that an interfering signal is introduced into the microphone signal when the mobile is transmitting. This interfering signal is transmitted along with the speech signal to the receiver. Due to the humming sound of the interfering signal it is commonly denoted the Bumblebee. The caller and the called party may also experience impairments introduced by the handset or environment, such as acoustic echo (see Table 1).

Table 1. Impairment Causes and Effects

\begin{tabular}{|c|c|c|}
\hline Problems & Identified Cause & Identified Effect \\
\hline $\begin{array}{l}\text { Background } \\
\text { Noise }\end{array}$ & $\begin{array}{l}\text { Noise from } \\
\text { equipment (trains, } \\
\text { airplanes) or people } \\
\text { in the background }\end{array}$ & $\begin{array}{l}\text { Caller has difficulty } \\
\text { hearing the called } \\
\text { party }\end{array}$ \\
\hline $\begin{array}{l}\text { Far-end } \\
\text { Noise }\end{array}$ & $\begin{array}{l}\text { Background noise in } \\
\text { the called party } \\
\text { Location }\end{array}$ & $\begin{array}{c}\text { Caller hears the } \\
\text { background noise } \\
\text { from the called party, } \\
\text { making conversation } \\
\text { difficult }\end{array}$ \\
\hline $\begin{array}{c}\text { Acoustic } \\
\text { Echo }\end{array}$ & $\begin{array}{l}\text { Sound reflections } \\
\text { from the caller } \\
\text { location are heard by } \\
\text { called party after } \\
\text { slight delay. }\end{array}$ & $\begin{array}{l}\text { Called party hears the } \\
\text { caller twice, with a } \\
\text { slight delay; often } \\
\text { interpreted as noise; } \\
\text { can be very annoying. }\end{array}$ \\
\hline $\begin{array}{c}\text { Level } \\
\text { Mismatch }\end{array}$ & $\begin{array}{l}\text { Networks or handsets } \\
\text { are not engineered to } \\
\text { proper levels; Callers } \\
\text { speaking unusually } \\
\text { loudly or softly. }\end{array}$ & $\begin{array}{l}\text { Caller or called party } \\
\text { sound too soft or } \\
\text { loud. }\end{array}$ \\
\hline
\end{tabular}

The present arrangement are likewise found to addresses the absence of an obligatory noise suppression technique in the GSM standard with a progressed ANC highlight that is a segment of a general VQA arrangement. VQA is regularly sent on the an interface, between the MSC and BSC, guaranteeing that all calls advantage from noise cancelation, paying little respect to handset sort and model.

\section{STANDARD TECHNIQUES}

A specific issue in a communication system is the generation of unwanted noise due to a number of reasons with the primary situation being is an impedance mismatch. when the four-wire network meets two-wired network mismatch in impedance will occur, this interface is also known as the Hybrid system. The acoustic noise is obtained, when an audio signal is vibrated in real surrounding, results the original signal is attenuated, time-delayed image of signal.

This study will concentrate on the event of acoustic noise, which is extremely normal in telecommunication frameworks. Figure.6 demonstrates a block diagram of adaptive noise cancellation method. Here the channel $\mathrm{H}(\mathrm{n})$ represent the impulsive reaction of the acoustic environment, W (n) represents the adaptive filter used to cancel the noise signal.

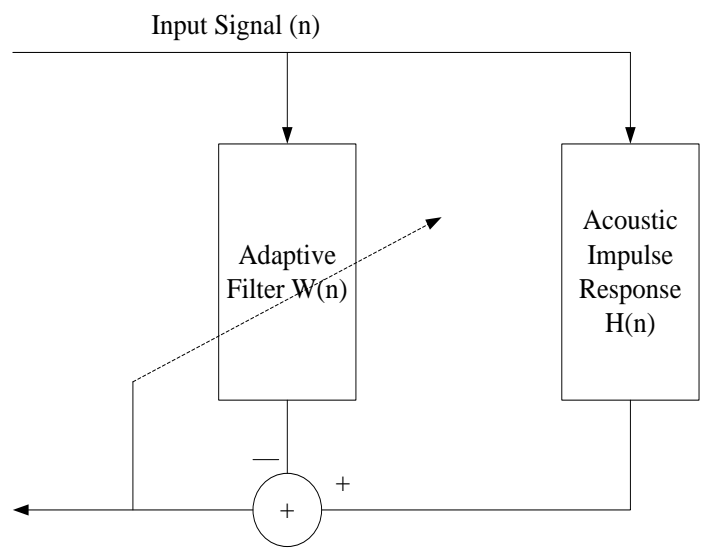

Fig.6. Adaptive echo Cancellation system

\subsection{First Technique}

The Adaptive Least Mean Square (LMS) technique was initially developed by Windrow and Hoff in the year 1959 [22] through their studies of an advance pattern recognition. An LMS algorithm are a type of adaptive filters and is use to impressionist a preferred filter by decided filter coefficients and that communicate to construct the least mean squares of error signal. The LMS algorithm is implemented and simulated using Matlab tool [23].

\subsection{2 Second Technique}

A sinusoid wave is be utilized as the test signal as a part of simulation and in analyses. The $\mu$ value in the LMS algorithm is an imperative quality in deciding the performance of echo cancelation. $\mu$ must be chosen somewhere around zero and double the converse rate of input power.

There are numerous algorithms that could be utilized as a part of noise cancelation or adaptive filtering; most are varieties of the least mean squared (LMS) algorithms. A few varieties included normalization LMS (NLMS) and the Recursive Least Squares algorithm (RLS). The LMS algorithm was selected because it is most generally utilized algorithm for noise removal problems. LMS is the most generally utilized algorithms and it is sufficiently powerful algorithm to finish the framework's prerequisites and is is simple algorithm compare to other algorithms. The LMS algorithm tries to minimize the excess MSE between the echo and its output. The adaptive LMS equation is given by:

$$
w(n+1)=w(n)+\mu * e(n) * x(n)
$$

where, where, $\mathrm{w}(\mathrm{n})$ is the present channel coefficients, $\mathrm{w}(\mathrm{n}+1)$ is future channel coefficients, $\mathrm{x}(\mathrm{n})$ are the input, $\mathrm{e}(\mathrm{n})$ is the error signal and $\mu$ is step size. $\mu$ decides the convergence or divergence and precision of adaptive filters. If $\mu$ is very large, the adaptive filter converges very fast, however could diverge if $\mu$ is too extensive. This excess of MSE may be undesirable to result. Similarly if $\mu$ is very small, filter will converge very slowly, which is proportionate to the algorithm having "long" memory and an undesirable quality. Each application will have an alternate step size that should be balanced. While picking the value of $\mu$, there should be balanced between convergence and the MSE. The value of $\mu$ will be chosen through experimentation so that speed at which the adaptive filter learns and the excess of 
MSE is acquired inside of utilization prerequisites. The value of $\mu$ varies from simulation and real time on account of the acquire contrasts between the systems.

\section{GSM NOISE MEASURING PRACTICES}

Current techniques are measuring speech quality in GSM systems; principally utilize drive test instruments in perspective of the perceptual assessment of speech quality systems [24], which is an objective procedure for assessing end-to-end voice quality composed by the International Telecommunication Union (ITU). Carriers regularly utilize PESQ with clean voice files to measure radio quality of their systems and to evaluate how debilitations identified with codec and frame losses effect listening quality. Another regular technique based on Ericsson's speech quality index, which regulates how codec and radio constraints like Bit error rate, frame ensure rate, discontinuous transmission and handover rates effect voice quality [25].

\subsection{Limitations of Conventional Speech- Quality}

\subsubsection{Measurement Techniques}

Basically, PESQ and SQI determine the effect of radio frequency (RF)-related losses on listening quality. In this way, they are not ready to catch and measure how other important speech quality impedances present in live callsdifferent background noise and acoustic noise echo are affects client experience. Large amounts of background noise are common in mobile calls, crowded places, environment etc. Acoustic sound is a nonlinear and obtained by low-end handsets due to lack of acoustic isolation between the speaker and the collector. Acoustic sound echo are also generated by Bluetooth devices and headsets, which are progressively increase of new laws that and drivers to utilize hands device, when talking on their telephones while driving. In addition, PESQ is a intrusive techniques, which implies that it can just quantify the nature of a predetermined number of test calls. It can't measure the quality of calls or the quality of calls set in areas, to those secured by the drive test routes. In addition to PESQ and SQI can just determines listening quality; they can't quantify conversational quality, which is significantly influenced by acoustic echo and transmission delay.

\subsection{Subjective Voice-Quality Test Methods}

Subjective methods use human listeners to measure voice quality so they can detect and evaluate all aspects of voice quality. The ITU has developed a number of recommendations for subjective testing, including the ITU Telecommunication Standardization Sector (ITU-T) P.800 [26], which defines the mean opinion score (MOS) as one important metric for subjective determination of transmission quality. The test subjects are asked to listen to prepared speech files and then determine the quality of the speech according to the following scale:

\section{- $\quad$ Excellent 5}

- $\quad$ Good 4

- $\quad$ Fair 3

- $\quad$ Poor 2

- $\quad$ Bad 1
The MOS is then calculated as an average of all participants' scores. The ITU-T P.835 recommendation [27] is particularly suitable for subjectively evaluating speech communications systems that include noise suppression algorithms. The scoring method is similar to ITU-T P.800 [26] with the addition that participants are asked to do three separate evaluations of the speech signal, the background signal, and the overall signal. A key limitation of these subjective test methods is that they are expensive, because they require a lot of testing time and a large number of participants. This means that carriers cannot employ these subjective methods for large-scale network testing and continuous monitoring of voice quality.

\subsection{Traditional Voice-Enhancement Solutions}

For many years, GSM carriers have used advanced drive test tools and methods to measure and mitigate radio networkrelated voice-quality impairments. Other important factors impacting voice quality in GSM networks are the so-called "external impairments"-background noise and nonlinear acoustic echo. It should be noted that, these external impairments are not being measured by traditional drive test tools. The impacts of background noise and nonlinear acoustic echo are therefore poorly understood in the GSM industry, and previous solutions addressing these impairments have not been optimal. There are several typical approaches to voice enhancement in GSM networks. Some GSM handsets have basic noise suppression and acoustic echo cancellation built in. However, the performance of these handset-based solutions varies considerably depending on handset vendor and model, so the GSM carrier cannot optimize its delivered quality and capacity in the way it could with network-based solutions that can process traffic from all handsets in the network. In addition, handsets have limitations in processing power and battery life that make it difficult and expensive to implement the most advanced noise-suppression and echocancellation algorithms. During the standardization of the latest codec in the GSM family, AMR, 3GPP identified the impact of background noise on voice quality, especially the limitations of the lowest AMR codec rates in noisy conditions. 3GPP issued a set of minimum performance requirements on handset based noise suppression in an attempt to address the problem [30]. Another approach is to build noise reduction into the codec itself. Unlike GSM, the CDMA standard has mandatory noise reduction integrated into the enhanced variable rate codec (EVRC) [31].

\section{RELATED WORK}

J. Bitzer and J. Rademacher [32] have proposed a two-stage technique. The principal step is to distinguish the disturbance signal and the second is to remove it. Because of the large variation of events over various recordings and small varieties for one recording they chose to utilize a single finger impression approach for identification. This implies that the user selects one typical burst and algorithm will locate every other occurrence. This type of signal's portion will be removed using cancellation techniques.

R. Ramesh et al. [33] have surmised a fundamental procedure that enterprises the abundance trademark in the interferer sign to cover it. The impedance concealment technique causes an all that much demonstrated a nonlinear bending to the 8PSK sign. This non-straight contortion is dealt with using a 
balanced equalizer that arrangements with a period changing info information joined with a lattice channel.

Raimund Meyer et al. [34] have introduced channel estimation and identification is examined for the MUROS downlink. Two novel channel estimation calculations are displayed, considering the specifics of the MUROS downlink. For location, a joint MLSE of both client signs can be connected if there should arise an occurrence of commotion restricted situations. For impedance constrained situations, things being what they are methodologies in light of the mono obstruction cancelation (MIC) calculation for single radio wire obstruction cancelation (SAIC) are better.

Jaymin Bhalani et al. [35] have considered nonlinear equalizer techniques like MLSE, Adaptive noise cancelation algorithms like LMS and RLS. The investigation of every schemes is given by their scramble plot by taking different regulation strategies and the general examination of method is confirmed for a few estimations of the SNR.

Jifeng Qin et al. [36] contain exhibit a transistor stage sketch of a covering modulator arrangement utilize a high good organization voltage double follow with a buck DC-DC converter for polar modulation. To accomplish a output voltage of the buck converter follow the Enhancement Datarate for GSM Evolution suggestion signal, current mode control and $10 \mathrm{MHz}$ frequency, and ripple cancellation circuits are built-in request to additional improve the spectral routine.

Jyrki T.J. Penttinen et al. [37] have demonstrated the behavior of the OSC capacity and radio performance as a function of the OSC penetration by comparing it with the GSM HR/FR traffic. The presented analysis is based on theoretical studies of the capacity and traffic behavior, as well as on single cell indoor measurements in the non-interfering environment. And they also presented, According to the analysis presented in this paper, the OSC might function in about $10 \%$ smaller coverage area than HR mode in a tightly dimensioned environment. This proportion depends on the network dimensioning, which should be taken into account in the interpretation of the presented results.

Hsiao-Bin Liang et al. [38] have illustrated the EM simulation by GEMS and real case verification by two way voice communication for single-end and differential layout architecture for immunizing the EMI noise from antenna's radiation. From GEMS's impulse simulation, the EMI noise voltage waveform is obviously degraded by common mode noise cancellation mechanism of differential architecture. From real case testing, they verify that the $217 \mathrm{~Hz}$ noise due to GSM TDMA switching disappears while differential architecture is adopted.

Such techniques have been utilized as a part of different applications, including communication frameworks, biomedical designing, and mechanical applications. Improvements in ALE in noise cancelation are investigated, including the principles, adaptive algorithms and recent changes on the filter design proposed to increase convergence rate and decrease the computational calculation for future execution.

Roshahliza M. Ramli et al. [39] have given a literature survey on Adaptive Line Enhancer (ALE) technique taking into account adaptive noise cancelation frameworks. Such strategies have been utilized as a part of different applications, including communication frameworks, biomedical and modern applications. Advancements in ALE in noise cancelation are investigated, including the standards, adaptive schemes and recent changes on the channel configuration proposed to expand the convergence rate and lessen the computational calculation and quality for future execution. The preferences and disadvantages of different adaptive filter algorithms like Least Mean Square, Recursive Least Square, and their variations, are reviewed.

\section{CONCLUSION FUTURE DIRECTION}

With a superior comprehension of the factors, affecting speech quality in their systems, GSM operators required to give a better equipped to keep up a good quality levels for their subscribers. Conventional quality management systems address an issue's portion, but the main issues are noise and nonlinear acoustic echo systems. Estimations of a large number of live GSM and CDMA calls show that ecological noise has a critical effect on customer experience. CDMA systems suppress from noise compare to GSM systems because of the inherent noise in CDMA. GSM carriers can address the issue of ecological noise by conveying a network based noise cancelation techniques. This paper has exhibited way different methodologies of LMS algorithms to remove echo. As a result of its effortlessness, the LMS algorithm is the most familiar adaptive noise cancellation algorithms. In any case, the LMS algorithm suffering from very slow and information dependent convergence behavior.

\section{REFERENCES}

[1] Vaseghi. S. V.2006. Advanced Digital Signal Processing and Noise Reduction. 3rd edition, Wiley publication

[2] Rossholm, A. 2006. On The Enhancement Of Audio And Video In Mobile Equipment. Blekinge Institute of Technology

[3] Gerald R.2002. McMenamin Forensic Linguistics: Advances in Forensic Stylistics, CRC Press, Law, pp. 360

[4] "Automatic gain control" $\left(22^{\text {nd }}\right.$ Sept, 2015)http://en.wikipedia.org/wiki/Automatic_gain_contr ol

[5] Joerg Bitzer and Jan Rademacher. (23nd Sept, 2015) Detection, Interpolation and Cancellation Algorithms for GSM burst Removal for Forensic Audio

[6] LTISystemTheory",http://en.wikipedia.org/wiki/LTI_sys tem_theoryKuldip Paliwal, Kamil Wo' jcicki. 2011. Benjamin Shannon, The importance of phase in speech enhancement, ScienceDirect-Elsevier, Speech Communication, Vol. 53, pp. 465-494

[7] Lim, J.S, A.V., Oppen Heim. (1979). Enhancement and bandwidth Compression of Noisy Speech, Proceedings of IEEE, vo.67, No.12

[8] Ekaterina Verteletskaya, Boris Simak. 2011. Noise Reduction Based on Modified Spectral Subtraction Method, International Journal of Computer Science, Vol. 38

[9] Phil S. Whitehead, David V. Anderson, Mark A. Clements. 2003. Adaptive, Acoustic Noise Suppression For Speech Enhancement, IEEE

[10] Joon-Hyuk Chang, Young-Joon Kim, Nam Soo Kim. 2000. Spectral Enhancement Based On Global Soft 
Decision, IEEE Signal Processing Letter, Vol.7, Iss.5, Pp. $108-110$

[11] Chabane Boubakir and Daoud Berkani, Chabane Boubakir and Daoud Berkani. 2010. Journal of Computer Science 6 (7): 700-705

[12] Olivier CappC. 1994. Elimination of the Musical Noise Phenomenon with the Ephraim and Malah Noise Suppressor, IEEE

[13] Anuradha R. Fukane, Shashikant L. Sahare. 2011. Different Approaches of Spectral Subtraction method for Enhancing the Speech Signal in Noisy Environments, International Journal of Scientific \& Engineering Research, Vol. 2, Issue. 5

[14] Guo Chen, Soo Ngee Koh, Ing Yann Soon.2003. Enhanced Itakura measure incorporating masking properties of human auditory system, Elsevier, Signal Processing, VOl. 83, pp. $1445-1456$

[15] J. Bobin1, Y. Moudden, J.-L. Starck and J. Fadili.2009. Sparsity and Morphological Diversity for Hyperspectral Data Analysis, IEEE

[16] Understanding the Echo "Phenomenon" Causes and Solutions. (Retrieved, 22 nd Oct, 2015). http://www.adaptivedigital.com/product/echo_cancel/ech o_explain.htm

[17] Datasheet of Detect networks, 2012

[18] Echo Explained. (23 $3^{\text {rd }}$ Oct, 2015). https://www.itu.int/rec/T-REC-G.107

[19] The Impact of Environmental Noise on Customer Experience in GSM and CDMA Networks. (2012). White paper, Detect Network

[20] 3GPP TR 06.78: Results of the AMR Noise Suppression Selection Phase

[21] 3GPP TS 26.077: Minimum Performance Requirements for Noise Suppressor.

[22] Least mean squares filter. (23 $3^{\text {rd }}$ Oct, 2015). http://en.wikipedia.org/wiki/Least_mean_squares_filter

[23] Oyerinde, O.O., and Mneney, S.H.2009. Variable Step Size Algorithms for Network Echo Cancellation, UbiCC Journal, Vol.4, No.3

[24] Scott Pennock.2002. Accuracy of the Perceptual Evaluation of Speech Quality (PESQ) algorithm, Proc. Of MESAQIN

[25] Optimizing Your GSM Network Today and Tomorrow Using Drive Testing to Estimate Downlink Speech Quality, Agilent, Application Note 1325

[26] Methods for subjective determination of transmission quality. $\left(23^{\text {rd }}\right.$, Oct, 2015). http://www.itu.int/rec/T-RECP.800-199608-I

[27] Subjective test methodology for evaluating speech communication systems that include noise suppression algorithm. (23 ${ }^{\text {rd }}$ Oct, 2015). http://www.itu.int/rec/TREC-P.835/en

[28] The E-model: a computational model for use in transmission 2015)https://www.itu.int/rec/T-REC-G.107

[29] About ETSI. (23 ${ }^{\text {rd }}$, Oct, 2015). http://www.etsi.org/about

[30] ANNEX C: Minimum Requirements for Mobile Networks and Terminals for the usage of the 'HD voice' logo Version 1.0, Retreived from www.gsma.com

[31] Enhanced Variable Rate Codec.(23 ${ }^{\text {rd }}$ Oct, 2015). http://en.wikipedia.org/wiki/Enhanced_Variable_Rate_C odecBitzer, Joerg, and Jan Rademacher.2003. Detection, Interpolation and Cancellation Algorithms for GSM burst Removal for Forensic Audio. Conference of the Int'l. Assoc. for Forensic Phonetics

[32] Ramesh, R.; Arslan, H.; Hafeez, A.; Hui, D.2009. Single Antenna Interference Cancellation for 8PSK Signals in EGPRS. Vehicular Technology Conference. VTC Spring IEEE 69th, pp.1-5,

[33] Meyer, R, Gerstacker, W.H, Obernosterer, F., Ruder, M.A., Schober, R.2009. Efficient receivers for GSM MUROS downlink transmission. Personal, Indoor and Mobile Radio Communications, IEEE 20th International Symposium, pp.2399-2403

[34] Bhalani, J., Trivedi, A.I., Kosta, Y.P.2009. Performance comparison of non-linear and adaptive equalization algorithms for wireless digital communication. Internet, 2AH-ICI First Asian Himalayas International Conference, pp.1-5

[35] Jifeng Qin; Rong Guo; Jinseok Park; Huang, A.2009.A low noise, high efficiency two stage envelope modulator structure for EDGE polar modulation," Circuits and Systems, IEEE International Symposium, pp.1089-1092

[36] Penttinen, J.T.J.; Calabrese, F.; Niemela, K.; Valerdi, D.; Molina, M.P.2010.Performance Model for Orthogonal Sub Channel in Noise-Limited Environment," Wireless and Mobile Communications (ICWMC), 6th International Conference, pp.56-61

[37] Hsiao-Bin Liang; Dau-Chyrh Chang; Guo-Hsiang Lo; Chin-Wen Chang.2011. The effect and solution of EMI caused by $217 \mathrm{~Hz}$ TDMA switching from compact size GSM security system. Electromagnetics, Applications and Student Innovation (iWEM), IEEE International Workshop, pp.186-190

[38] Roshahliza M. Ramli, Ali O. Abid Noor, and Salina Abdul Samad. 2012. A Review of Adaptive Line Enhancers for Noise Cancellation", Australian Journal of Basic and Applied Sciences, Vol.6 (6), pp. 337-352

[39] Ali O. Abid Noor, Salina Abdul Samad and Aini Hussain.2012. Development of a Voice Activity Controlled Noise Canceller. Open Access sensors, pp. 6727-6745 